\title{
Hipertensión Arterial: Puntos relevantes del VI reporte del comité Americano sobre Prevención, Detección, Evaluación y Tratamiento (JNC VI)
}

The sixth report of the Joint National Committee on Prevention, Detection, Evaluation and Treatment of High Blood Pressure (JNC VI). Arch Intern Med 1997;157:2413-2445

Dra. Nora Gimpel

Introducción

El diagnóstico, tratamiento y control de la hipertensión arterial (HTA) ha mejorado en las últimas décadas. Desde la publicación de 5to Reporte en EE.UU ha disminuido la morbimortalidad por accidente cerebrovascular (ACV) y enfermedad coronaria atribuible a la HTA a la vez que ha se ha incrementado la incidencia de insuficiencia cardíaca y insuficiencia renal. Estos datos y la alta prevalencia de la HTA (25\% en la población general) (2) han determinado la publicación de esta nueva guía basada en el minucioso análisis de los estudios que proveen evidencia para determinar recomendaciones en el diagnóstico, la prevención y el tratamiento de la HTA.

El objetivo del VI reporte es ofrecer una guía para ser utilizada por el médico en el manejo de la HTA. Los desafíos propuestos son: prevenir el aumento de la incidencia de HTA con la edad; disminuir la prevalencia de HTA; aumentar la detección y mejorar el control; reducir los riesgos cardiovasculares; mejorar el reconocimiento de la HTA normal-alta; reducir las variaciones según región y nivel socioeconómico; mejorar las posibilidades de tratamiento y estimular programas comunitarios.

Medición, clasificación y rastreo de la Tensión Arterial (TA)

- Para la toma de TA el paciente debe permanecer en.reposo por 5 minutos y no debe haber fumado o ingerido cafeína en los 30 minutos previos al registro. Debe estar sentado con`el brazo descubierto, apoyado y a la altura del corazón. La cámara del manguito debe tener una longitud de $2 / 3$ del brazo.

- La TA debe ser registrada preferentemente con un esfigmomanómetro de mercurio, un manómetro aneroide o un dispositivo electrónico calibrado y validado. Deberá registrarse la tensión arterial sistólica (TAS) y diastólica (TAD). La desaparición del sonido (fase 5) define la TAD. Se deben promediar dos o más lecturas separadas por 2 minutos, y si estas difieren en más de $5 \mathrm{mmHg}$ deben obtenerse nuevos registros y promediarlos.

Clasificación actual deTensión arterial en adultos $>$ de 18 años

\begin{tabular}{|l|ccc|}
\hline Categoría & \multicolumn{1}{|c}{ TAS } & & TAD \\
\hline Optima & $<120$ & y & $<80$ \\
\hline Normal & $<130$ & y & $<85$ \\
\hline Normal alta & $130-139$ & 0 & $85-89$ \\
\hline Estadio 1 & $140-159$ & 0 & $90-99$ \\
\hline Estadio 2 & $160-179$ & 0 & $100-109$ \\
\hline $\begin{array}{l}\text { Estadio 3 } \\
\text { (reune estadios 3 y 4 de } \\
\text { la clasificación anterior) }\end{array}$ & $\geq 180$ & 0 & $\geq 110$ \\
\hline
\end{tabular}

Registro inicial de TA y seguimiento

\begin{tabular}{|c|c|c|}
\hline TAS & TAD & Seguimiento recomendado \\
\hline$<130$ & $<85$ & Control cada 2 años \\
\hline $130-139$ & $85-89$ & Control anual \\
\hline $140-159$ & $90-99$ & Confirmar dentro de los 2 meses \\
\hline $160-179$ & $100-109$ & Evaluar o iniciar terapéutica dentro del mes \\
\hline$\geq 180$ & $\geq 110$ & $\begin{array}{c}\text { Evaluar o iniciar terapéutica inmediatamente o en } \\
\text { la semana dependiendo de la situación clínica }\end{array}$ \\
\hline
\end{tabular}

- El diagnóstico debe basarse en el promedio de 2 o más registros tomados en 2 o más visitas luego del rastreo.

- Si la TAS y TAD entran en distintas categorías se debe considerar la categoría más alta para clasificar el estado de la TA del paciente (Ej. 160/92 Estadío 2, 175/120 Estadío 3).

- La hipertensión sistólica aislada es definida por TAS $\geq 140$ y TAD $<90$.

Otras consideraciones:

El monitoreo automático ambulatorio de la TA (presurometría de 24hs. o Holter de TA) no se recomienda como evaluación de rutina. Puede ser considerado de utilidad en: pacientes con sospecha de HTA de consultorio o guardapolvo blanco, evaluación de resistencia a drogas, síntomas de hipotensión con antihipertensivos y síndromes sincopales.

\section{Evaluación inicial del paciente hipertenso}

La evaluación del paciente con HTA tiene 3 objetivos:

1. Identificar causas conocidas de HTA

2. Evaluar la presencia o ausencia de daño de órganos blanco,

3. Identificar otros factores de riesgo cardiovasculares y comorbilidades que puedan definir el pronóstico y tratamiento.

Otros estudios están indicados en casos donde la edad ( $<25$ o $>50$ años), la historia, el examen físico, los estudios iniciales o la severidad de la HTA sugieren otras causas así como en aquellos pacientes con fallas al tratamiento apropiado, en estadío 3 de HTA o comienzo brusco de la HTA. 


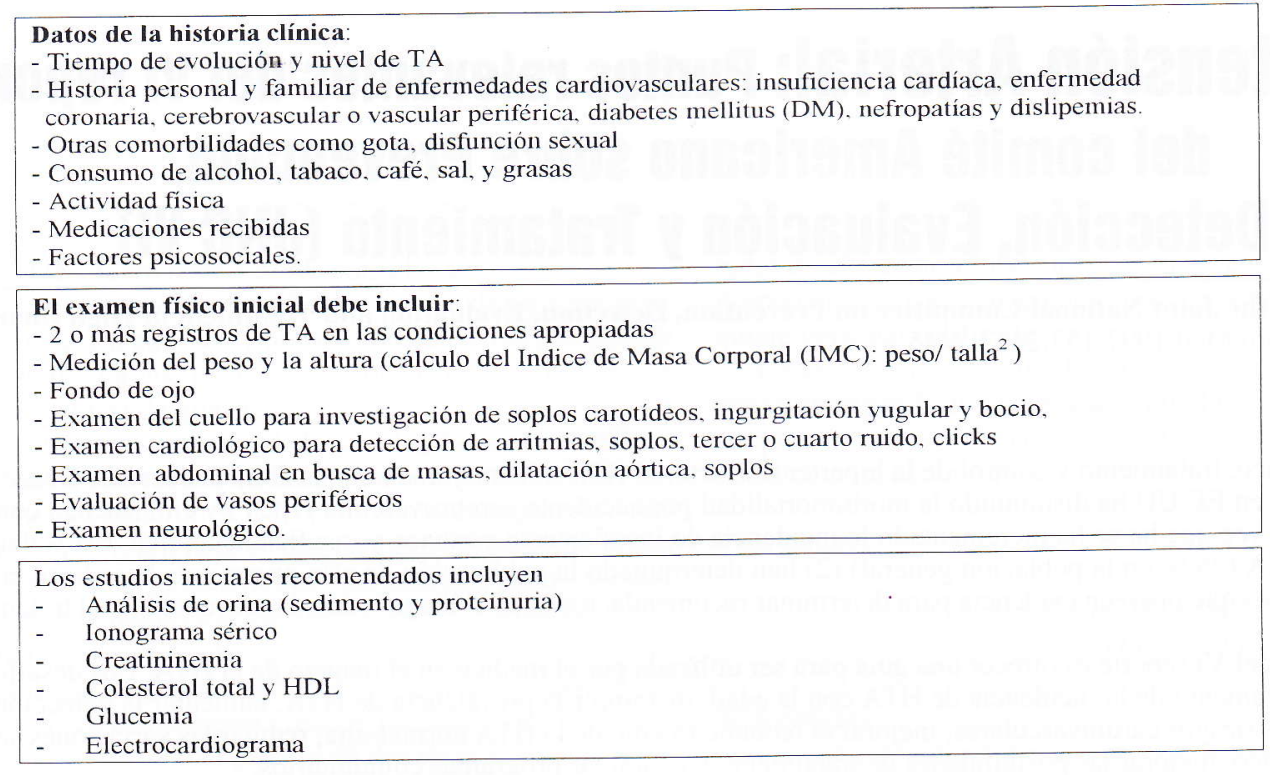

\section{Estratificación del riesgo}

El riesgo de enfermedad cardiovascular en pacientes hipertensos está determinada no solo por el nivel de TA sino también por la presencia o ausencia de daño en órganos blanco y otros factores asociados. El JNC VI propone una estratificación de riesgo con tres categorías. Las recomendaciones de las distintas estrategias de intervención enfatizan los riesgos y beneficios absolutos más que los relativos (aquellos pacientes con mayor riesgo basal lograrán mayores beneficios).

\begin{tabular}{|c|c|}
\hline \multicolumn{2}{|c|}{ Clasificación de riesgos en pacientes con hipertensión } \\
\hline Riesgos mayores (RM) & $\begin{array}{c}\text { Daño órgano blanco (DOB) } \\
\text { Enfermedad cardiovascular }(\mathrm{ECV})\end{array}$ \\
\hline $\begin{array}{l}\text { - Tabaquismo } \\
\text { - Dislipemia } \\
\text { - Diabetes } \\
\text { - Edad >60 años } \\
\text { - Sexo masculino o mujer posmenopáusica } \\
\text { - Historia familiar de ECV: mujeres }<65 \text {, } \\
\text { hombres }<55\end{array}$ & $\begin{array}{l}\text { - Hipertrofia ventricular izquierda } \\
\text { - Historia de infarto agudo de miocardio } \\
\text { (IAM) o angor } \\
\text { - Historia de revascularización coronaria } \\
\text { - Insuficiencia cardíaca } \\
\text { - ACV o ataque isquémico transitorio } \\
\text { - Nefropatía } \\
\text { - Vasculopatía periférica } \\
\end{array}$ \\
\hline
\end{tabular}

Según estos factores de riesgo y el estadio de HTA se establecieron los distintos grupos de riesgo y las siguientes recomendaciones de tratamiento:

\begin{tabular}{|l|c|c|c|}
\hline \multicolumn{1}{|c|}{ Estadio de HTA } & $\begin{array}{c}\text { Grupo A } \\
\text { No RM, No DOB ni ECV }\end{array}$ & $\begin{array}{c}\text { Grupo B } \\
\text { o más RM (no DM), } \\
\text { No DOB ni ECV }\end{array}$ & $\begin{array}{c}\text { Grupo C } \\
\text { DOB / ECV y/o DM, } \\
\text { con o sin otros RM }\end{array}$ \\
\hline TA Normal -alta & $\begin{array}{c}\text { Modificación del estilo de } \\
\text { vida }\end{array}$ & $\begin{array}{c}\text { Modificación del estilo de } \\
\text { vida }\end{array}$ & $\begin{array}{c}\text { Terapia con drogas } \\
\text { Estadio 1 }\end{array}$ \\
$\begin{array}{c}\text { Modificación del estilo de } \\
\text { vida (1 año) }\end{array}$ & $\begin{array}{c}\text { Modificación del estilo de } \\
\text { vida (6 meses) }\end{array}$ & Terapia con drogas \\
\hline Estadio 2 y 3 & Terapia con drogas & Terapia con drogas & Terapia con drogas \\
\hline
\end{tabular}

Esta clasificación es una de las herramientas más prácticas de este Reporte. Está dirigida a identificar el riesgo del paciente (evaluación inicial) y planificar la estrategia de tratamiento de acuerdo al grupo al que pertenece. Por ejemplo, en un paciente con TA normalalta e insuficencia cardíaca, o DM o nefropatía se deberá considerar la terapeútica con drogas inmediatamente; y un paciente con TA normal-alta o estadio 1 , sin factores de riesgo, ni enfermedad cardiovascular ni daño de órgano blanco debe iniciar un programa de modificación del estilo de vida (medidas no farmacológicas). Es importante mencionar que las medidas no farmacológicas deben recomendarse siempre, aún con los tratamientos farmacológicos.

\section{Metas del tratamiento}

La principal meta es reducir la morbi-mortalidad asociada a la HTA. Esto significará lograr y mantener la TAS $<140 \mathrm{mmHg}$ y TAD $<90$ $\mathrm{mmHg}$ (registros de consultorio) o $<130 / 85 \mathrm{mmHg}$ (registros fuera del consultorio). En pacientes con HTA sistólica o pacientes ancianos, la TAS $<160 \mathrm{mmHg}$ resultará aceptable. 
En pacientes diabéticos la meta es $\mathrm{TA}<130 / 85 \mathrm{mmHg}$.

Modificaciones del estilo de vida (medidas no farmacológicas)

Estas medidas deberían alentarse en la mayoría de los pacientes con HTA leve, especialmente si no tienen factores de riesgo cardiovascular asociados o evidencias de daño de órgano blanco. Todos los pacientes hipertensos deben ser estimulados a perpetuar éstas modificaciones como para, de necesitar fármacos, poder disminuir el número o dosis de drogas antihipertensivas necesarias.

- Reducción de peso: En los individuos obesos o con sobrepeso (IMC $\geq 27$ ), y pacientes hipertensos ancianos la intervención no farmacológica ha demostrado importantes beneficios. El exceso de grasa en la región superior del cuerpo (visceral o abdominal) evidenciado por una circunferencia de cintura de $85 \mathrm{~cm}$ o más en la mujer y $98 \mathrm{~cm}$ o más en los hombres está asociada a un aumento del riesgo de HTA, DM y mortalidad cardiovascular. La reducción de sólo $3 \mathrm{~kg}$. de peso produce una caída de 7 y $4 \mathrm{~mm} \mathrm{Hg}$ mientras que la reducción de $12 \mathrm{~kg}$. produce una caída de $21 / 13 \mathrm{~mm} \mathrm{Hg}$ en la TAS y la TAD, respectivamente.

- Restricción de alcohol: El consumo excesivo de alcohol es un importante factor de riesgo asociado a la HTA y a ACV y puede causar resistencia a fármacos antihipertensivos. Se recomienda un consumo diario máximo de $28 \mathrm{~g} \mathrm{(} 30 \mathrm{ml}$ ) de alcohol (Ej: $750 \mathrm{ml}$ de cerveza, $300 \mathrm{ml}$ de vino, $60 \mathrm{ml}$ de bebidas de alta graduación).

- $\quad$ Ejercicio: El ejercicio aeróbico regular puede facilitar la disminución de peso, mejorar la capacidad funcional y reducir otros factores de riesgo cardiovasculares. La TA puede disminuir con un programa graduado de actividad física como 30-45 minutos diarios de caminatas enérgicas 3 a 4 veces por semana. Los ejercicios isométricos (Ej: complemento de pesas, etc. ) no reemplazan a los aeróbicos y pueden producir incremento de la TA.

- Restricción de sal: La asociación positiva entre el consumo de sal y el nivel de TA ha sido demostrada. Los efectos más importantes se observaron en pacientes ancianos y con cifras elevadas de TA. Algunos estudios observacionales y controlados sugieren que la reducción de sodio de la dieta podría asociarse a la reducción en la terapeútica farmacológica antihipertensiva, protección de osteoporosis, y regresión de hipertrofia ventricular izquierda. La recomendación de una dieta con $2.4 \mathrm{~g}$ de sodio/día $(6 \mathrm{~g} \mathrm{Cl} \mathrm{Na})$ parece ser actualmente la más apropiada y los pacientes que más se benefician son los pacientes con historia familiar de HTA, TA inicial más elevada y diagnóstico luego de los 40 años.

- Otros factores dietéticos: El alto contenido de Potasio en la dieta puede proteger contra el desarrollo y el control en pacientes hipertensos. De todos modos, la ingesta de potasio $(90 \mathrm{mmol} /$ día) debe mantenerse preferentemente con fuentes alimentarias como frutas, vegetales, productos hipograsos y sustitutos de la sal. Estos últimos son especialmente beneficiosos en pacientes que reciben diuréticos, pero deben ser utilizados con precaución en los que reciben ahorradores de potasio e inhibidores de la enzima convertidora de angiotensina (IECA). El aumento de Calcio y magnesio en la dieta puede disminuir la TA en algunos pacientes con HTA, pero este beneficio es mínimo; por lo tanto se recomienda mantener una adecuada ingesta de calcio y no administrar suplementos. Resulta beneficio el tratamiento dietético o farmacológico de las dislipemias. Los estudios controlados con variaciones del total de grasas de la dieta y la proporción de grasas saturadas e insaturadas han tenido poco efecto en la TA. Un reciente estudio controlado ha demostrado que la dieta rica en frutas y verduras tiene efecto hipotensor en pacientes normotensos e hipertensos.

- Otras medidas: El tabaquismo es un importante factor de riesgo cardiovascular por lo tanto resulta apropiado estimular el abandono del tabaco. La cafeína puede producir aumentos temporarios de la TA, pero no existe evidencia para limitar su consumo en los hipertensos a menos que produzca efectos adversos cardiovasculares como palpitaciones. El rol de técnicas en el manejo del stress y la HTA aún no está determinado y por el momento no se recomienda.

\section{Medidas Farmacológicas}

Una enorme cantidad de fármacos antihipertensivos están aprobados para reducir la TA: Diuréticos, beta bloqueantes, alfa agonistas, alfa bloqueantes, antagonistas cálcicos, IECA, antagonistas del receptor de la angiotensina II (losartan, valsartan), agentes periféricos (reserpina), vasodilatadores directos (hidralazina, minoxidil), combinaciones alfa y beta bloqueantes (carvedilol, labetalol) y asociaciones (beta bloquentes- diuréticos; IECA- diuréticos).

Las siguientes son algunas consideraciones relevantes al momento de decidir el tratamiento farmacológico del paciente hipertenso:

- Comenzar la terapia farmacológica antihipertensiva con diuréticos o beta bloqueantes si no existe la indicación específica para usar otra droga. Es la estrategia con mayor evidencia a su favor para disminuir la morbi-mortalidad (grado I*).

- $\quad$ Resulta apropiado comenzar con dosis bajas del fármaco elegido (Ej. hidroclorotiazida 12.5mg,día) y modificarla según la edad, necesidad y respuesta del paciente. La formulación óptima debe proveer eficacia por $24 \mathrm{hs}$. en una o dos dosis diarias, para aumentar la la adherencia y ofrecer el control persistente de la TA a costos adecuados.

- Algunas recomendaciones de fármacos son más específicas como

$\Rightarrow \quad$ DM con proteinuria o microalbuminuria: IECA

$\Rightarrow \quad$ Insuficiencia cardíaca: IECA o diuréticos

$\Rightarrow \quad$ IAM: beta bloqueantes o IECA (si existe disfunción

sistólica)

$\Rightarrow \quad$ HTA sistólica: diuréticos o antagonistas cálcicos de larga duración

$\Rightarrow \quad$ Angor: beta bloqueantes o antagonistas cálcicos

$\rightarrow \quad$ Osteoporosis: tiazidas.

- Algunos fármacos deben utilizarse con cuidado en pacientes hipertensos con comorbilidades:

a) Broncoespasmo: beta bloqueantes

$\Rightarrow \quad$ Depresión: beta bloqueantes, alfa agonistas centrales

a) Gota:diuréticos 
c) DM: beta bloqueantes

c Enfermedad renovascular: IECA, bloq. angiotensina II. pendiente.

Algunas asociaciones con dosis bajas de dos agentes pueden ofrecer beneficio minimizando los efectos adversos dosis-de-

Los antagonistas del receptor de la angiotensina II producen efectos similares a los IECA, evitando los efectos adversos más comunes (tos). Ante la ausencia de datos que documenten igual protección renal y cardíaca a largo plazo, deben utilizarse sóla en quienes los IECA están indicados y no han sido tolerados.

- Estudios de prevención secundaria de enfermedad coronaria han sugerido la posibilidad de efectos perjudiciales en el caso de antagonistas cálcicos de acción corta como la nifedipina. Otro estudio también sugiere un aumento de la morbilidad cardiovascular (mayor incidencia de IAM) en hipertensos tratados con bloqueantes cálcicos de acción corta incluyendo el diltiazem y el verapamilo. Estas drogas no debieran considerarse como primera línea de tratamiento farmacológico en HTA no complicada.

- Los pasos sugeridos para el tratamiento antihipertensivo son:

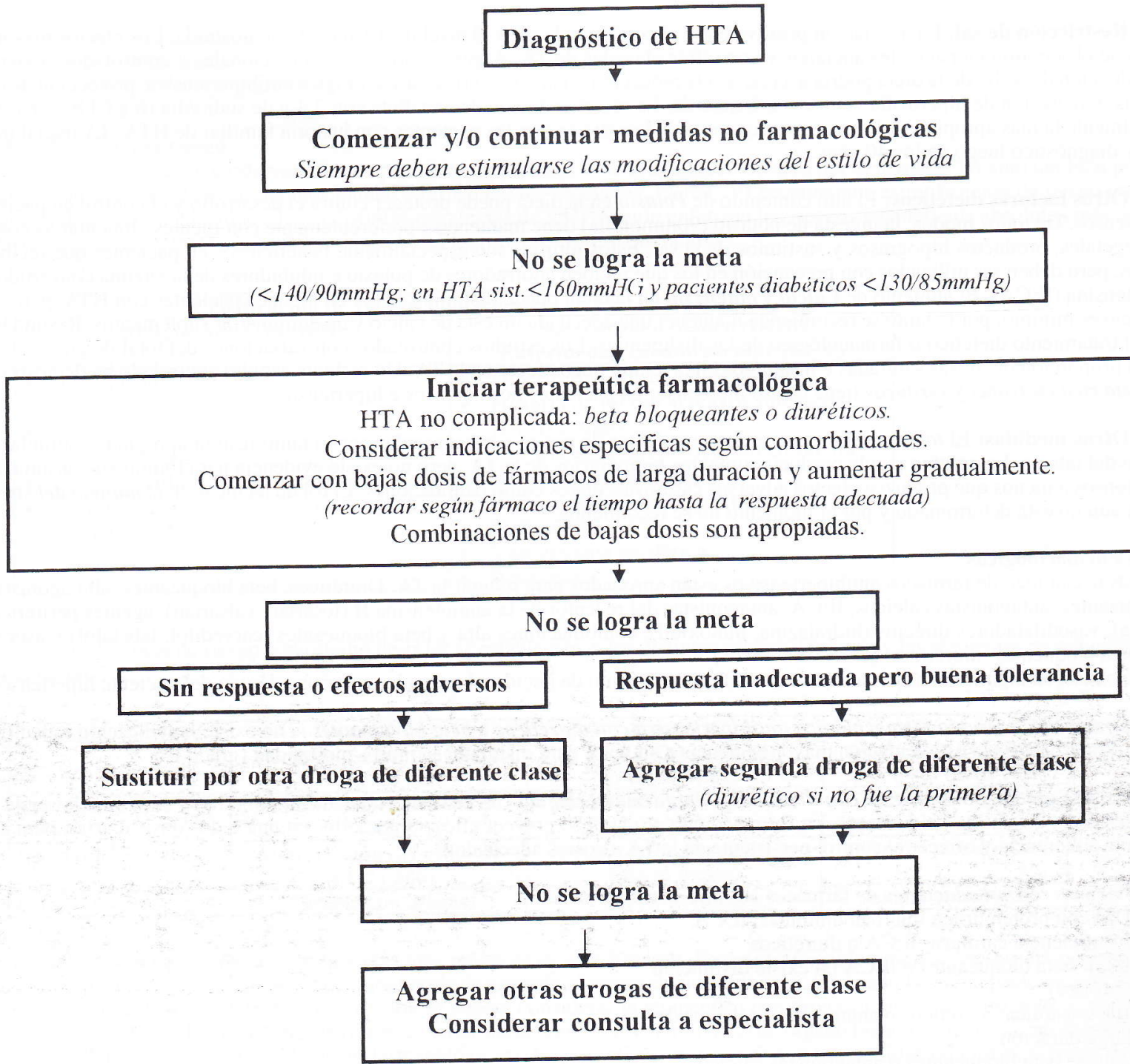

\footnotetext{
* Ver Glosario
}

Nota: Esta actualización destaca los puntos más sobresalientes del VI Reporte del JNC y no debe considerarse una revisión sistemática del tema hipertensión. Dado el elevado número de referencias contenidas en el reporte original remitimos al lector al mismo si desea obtener las citas bibliográficas. 